\title{
Isolation and identification of alkaloids and anthocyanins from flower and bulb of Lycoris radiata using HPLC and LC-ESI-MS
}

\author{
Jin-Hyuk Chun ${ }^{1}$, In Hyuk Jang ${ }^{2}$, Mariadhas Valan Arasu ${ }^{1}$, Naif Abdullah Al-Dhabi ${ }^{3}$, \\ Veeramuthu Duraipandiyan ${ }^{3}$, Dong-Hoon Lee ${ }^{4}$, Sanghyun Lee ${ }^{5}$, Sun-Ju Kim ${ }^{1^{\star}}$ \\ ${ }^{1}$ Department of Bio-Environmental Chemistry, Chungnam National University, Daejeon, Republic of Korea; ${ }^{*}$ Corresponding Author: \\ kimsunju@cnu.ac.kr \\ ${ }^{2}$ Department of Formulation, Sungbo Chemicals Co., Ltd., Goyang-si, Republic of Korea \\ ${ }^{3}$ Department of Botany and Microbiology, College of Science, King Saud University, Riyadh, Saudi Arabia \\ ${ }^{4}$ Yeonggwang Agricultural Technology Center, Yeonggwang-Gun, Republic of Korea \\ ${ }^{5}$ Department of Integrative Plant Science, Chung-Ang University, Anseong, Republic of Korea
}

Received 20 November 2012; revised 25 December 2012; accepted 8 January 2013

\begin{abstract}
Three anthocyanins (cyanidin 3-diglucoside, cyanidin 3-sambubioside and cyanidin 3-glucoside) together with eleven known alkaloids (lycoricidine, hipppeastrine, O-Demethyllycoramin, lycoricidinol, galanthine, lycorine, lycorenine, lycoramine, galanthamine, homolycorine and pretazettine) were identified in the flower and bulb of Lycoris radiata using high-performance liquid chromatography coupled with UV detection and electrospray ionization mass spectrometry. Anthocyanins play a major role in protecting plant's DNA from the UV spectrum of sunlight and also in attracting insects for the purpose of pollination. Thus, knowledge on the contents and types of anthocyanins of $L$. radiata will help to evaluate the adaptive evolution of flowers and provide useful information for the ornamental breeding.
\end{abstract}

Keywords: Lycoris radiata; Anthocyanins;

Alkaloids; Flower; Bulb

\section{INTRODUCTION}

Plants of the family Amaryllidaceae are well known for their ornamental values and also for their alkaloids they produce. Some of these alkaloids exhibit interesting pharmacological and biological activities. Lycoris radiata belongs to Amaryllidaceae family of perennial plants, and 20 different species are distributed in East Asia including China, Japan, Korea and Nepal. The Korean peninsula has seven Lycoris species such as L. flavescens, $L$. chinensis var. sinuolata, $L$. uydoensis, $L$. sanguinea var. koreana, L. chejuensi, L. radiata and L. squa- migera respectively [1]. Since 2000, several Korean researches have reported identification of metabolites from Lycoris species [2,3]. L. radiata has ornamental and medical values and therefore the interest of plant breeding is high. Floral colors of $L$. radiata are due to biological pigments of different kinds of molecules, such as porphyrins, carotenoids, anthocyanins and betalains. Anthocyanins occur only in terrestrial plants and are thought to be evolutionarily adaptive for several different plants biological processes. Four anthocyanin components, pelargonidin3-glucosides, pelargonidin-3-xylosylglucosides, cyanidin3-glucosides, and cyanidin-3-xylosylglucosides were identified in L. radiata [4]. To our best knowledge, cyanidin 3diglucoside and cyanidin 3-sambubioside have not been reported in the flower of $L$. radiata, but they were found to be an important determinant of floral colors in L. radiata.

\section{MATERIALS AND METHODS}

\subsection{Plant Materials}

Flowers and bulbs of $L$. radiata harvested in 2011 were provided by the Yeonggwang Agricultural Technology Center (Yeonggwang-gun, Korea).

\subsection{Extraction of Alkaloids and Anthocyanins}

For alkaloids, a $100 \mathrm{mg}$ of lyophilized powder was transferred to $2 \mathrm{~mL}$ eppendorf tube and mixed with $1 \mathrm{~mL}$ of water/sulfuric acid solution $(99: 1, \mathrm{v} / \mathrm{v})$. The solution was mixed thoroughly by vortexing for 2 min and then allowed to stand at room temperature for $24 \mathrm{~h}$ without disturbance. In the case of anthocynins, a $100 \mathrm{mg}$ portion of lyophilized powder was transferred to a $2 \mathrm{~mL}$ eppendorf tube, and $2 \mathrm{~mL}$ of water/formic acid solution (95:5, 
v/v) was added. The solution was vigorously mixed for 5 min, sonicated for $20 \mathrm{~min}$, and centrifuged at $8000 \mathrm{rpm}$ for $30 \mathrm{~min}$. The extract was filtered through a $0.45 \mu \mathrm{m}$ PTFE syringe filter (Advantec DISMIC-13HP, Toyo Roshi Kaisha, Ltd., Tokyo, Japan). The filtrate was analyzed by HPLC and LC-ESI-MS.

\subsection{LC-ESI-MS Analysis of Alkaloids and Anthocyanins}

HPLC analyses were carried out with a flow rate of $1.0 \mathrm{~mL} / \mathrm{min}$ at column oven temperature of $40^{\circ} \mathrm{C}$ and detector wavelength of $292 \mathrm{~nm}$. The solvent system employed was (A) $10 \mathrm{mM}$ ammonium carbonate and (B) $10 \% 10 \mathrm{mM}$ ammonium carbonate in acetonitrile. The solvent program used is as follows: 0 min solvent $\mathrm{B} 0 \%$, 13 min solvent B 7\%, then kept constant at solvent B 7\% till $18 \mathrm{~min}, 23 \mathrm{~min}$ solvent B 10\%, then kept constant at solvent B 10\% till $28 \mathrm{~min}, 35 \mathrm{~min}$ solvent B 20\%, 50 min solvent B 70\%, then kept constant at solvent B 70\% till $55 \mathrm{~min}$, rapidly reduce solvent $\mathrm{B}$ to $0 \%$ at $55.1 \mathrm{~min}$, and then kept constant at solvent B $0 \%$ till total $60 \mathrm{~min}$. For the identification of individual alkaloids, the MS analysis was carried out with an ESI interface operating in the positive ion mode $[\mathrm{M}+\mathrm{H}]^{+}$. The MS operating conditions were as follows: ion spray voltage, $5.5 \mathrm{kV}$; curtain gas $\left(\mathrm{N}_{2}\right)$, 20 psi; nebulizing gas and heating gas $\left(\mathrm{N}_{2}\right)$, $50 \mathrm{psi}$; heating gas temperature, $550^{\circ} \mathrm{C}$; spectra range, $\mathrm{m} / \mathrm{z} 100$ - 500 (scan time, $4.8 \mathrm{sec}$ ).

For anthocynins, an API 4000 Q TRAP tandem mass spectrometer (Applied Biosystems, Foster City, CA), equipped with an Agilent 1200 series HPLC system and an electrospray ionization-tandem mass spectrometry (ESI-MS) source in positive ion mode was used. The HPLC conditions were the same as those described above, but the mobile phase was changed to (A) water/ formic acid (99:1, v/v) and (B) acetonitrile/formic acid $(99: 9, \mathrm{v} / \mathrm{v})$ because of low $\mathrm{pH}$ of less than 2.0 required.

\section{RESULTS AND DISCUSSION}

The MS spectral data by comparing with data of a previous report $[5,6]$ led to identification of eleven known alkaloids (lycoricidine (peak no. 4), hipppeastrine (6), O-demethyllycoramine (7), lycoricidinol (8), galanthine (9), lycorine (10), lycorenine (12), lycoramine (13), gaanthamine (14), homolycorine (16), and pretazettine (18). Seven unknown compounds were also observed. The peak numbers are the elution order in the HPLC chromatogram in Table 1. LC-ESI-MS spectra of lycorine and cyanidin 3-sambubioside (anthocyanin) were showed in Figure 1 and Figure 2(a). Identification of anthocyanins was primarily based on the comparison of their retention time and elution order together with mass spectrometric data with standards and isolation methods de- scribed previously $[7,8]$. Separation of identified anthocyanins on HPLC with their retention time and elution order together with mass spectrometric data were shown in Table 1, Figure 2(b) and Figure 3. LC-ESI-MS spectra of three anthocyanins were compared with the published reports of other anthocyanins derivatives, which coincide in fragmentation patterns and further confirmed that the identified anthocyanins were cyanidin 3-diglucoside, cyanidin 3-sambubioside and cyanidin 3-glu-coside respectively. HPLC chromatogram of peaks 1 and 3 had exhibited similar MS profiles but showed different retention times $(23.8$ and $26.0 \mathrm{~min}$ ). The LC-ESI-MS analysis indicated that peaks 1,2 and 3 contained aglycone cyanidin (MW, molecular weight, 287) as a common backbone with varied monosaccharide glucose (MW, 180) and peaks 2 had monosaccharide with the combination of D-xylose and D-glucose (MW, 312). Cyanidin 3-diglucoside and cyanidin 3-glucoside exhibited molecular ions at $\mathrm{m} / \mathrm{z} 287$, respectively, whereas cyanidin 3-sambubioside showed at m/z 294 because this compound produced only one major fragment $[9,10]$. Peaks 1 and 3 had the same aglycone (cyanidin) as peak 3 but

Table 1. Alkaloids identified in the bulb of Lycoris radiate.

\begin{tabular}{|c|c|c|c|c|}
\hline No. ${ }^{a}$ & $\mathrm{RT}^{\mathrm{b}}$ & $\begin{array}{l}\text { Trivial } \\
\text { names }\end{array}$ & {$[\mathrm{M}+\mathrm{H}]^{+}(\mathrm{m} / \mathrm{z})$} & $\begin{array}{l}\text { Fragment } \\
\text { ion }(\mathrm{m} / \mathrm{z})\end{array}$ \\
\hline 1 & 12.13 & Unknown & 279 & $290 / 326$ \\
\hline 2 & 12.85 & Unknown & 301 & $279 / 264$ \\
\hline 3 & 14.99 & Unknown & 268 & $278 / 286$ \\
\hline 4 & 25.44 & Lycoricidine & 292 & $279 / 228$ \\
\hline 5 & 29.56 & Unknown & 290 & $272 / 254$ \\
\hline 6 & 30.72 & Hipppeastrine & 316 & $334 / 288$ \\
\hline 7 & 33.11 & $O$-Demethyllycoramine & 276 & $290 / 219$ \\
\hline 8 & 35.79 & Lycoricidinol & 308 & $290 / 276$ \\
\hline 9 & 39.10 & Galanthine & 318 & $279 / 276$ \\
\hline 10 & 39.59 & Lycorine & 288 & $332 / 270$ \\
\hline 11 & 45.98 & Unknown & 480 & $428 / 279$ \\
\hline 12 & 46.70 & Lycorenine & 318 & $300 / 288$ \\
\hline 13 & 46.99 & Lycoramine & 290 & $233 / 215$ \\
\hline 14 & 48.09 & Galanthamine & 288 & $213 / 231$ \\
\hline 15 & 48.46 & Unknown & 302 & $284 / 255$ \\
\hline 16 & 48.74 & Homolycorine & 316 & $302 / 298$ \\
\hline 17 & 51.11 & Unknown & 256 & $210 / 137$ \\
\hline 18 & 53.87 & Pretazettine & 332 & $272 / 240$ \\
\hline
\end{tabular}

${ }^{\mathrm{a}}$ The elution order of alkaloids in HPLC chromatogram (Figure 1); ${ }^{\mathrm{b}}$ Retention time ( $\min )$ 


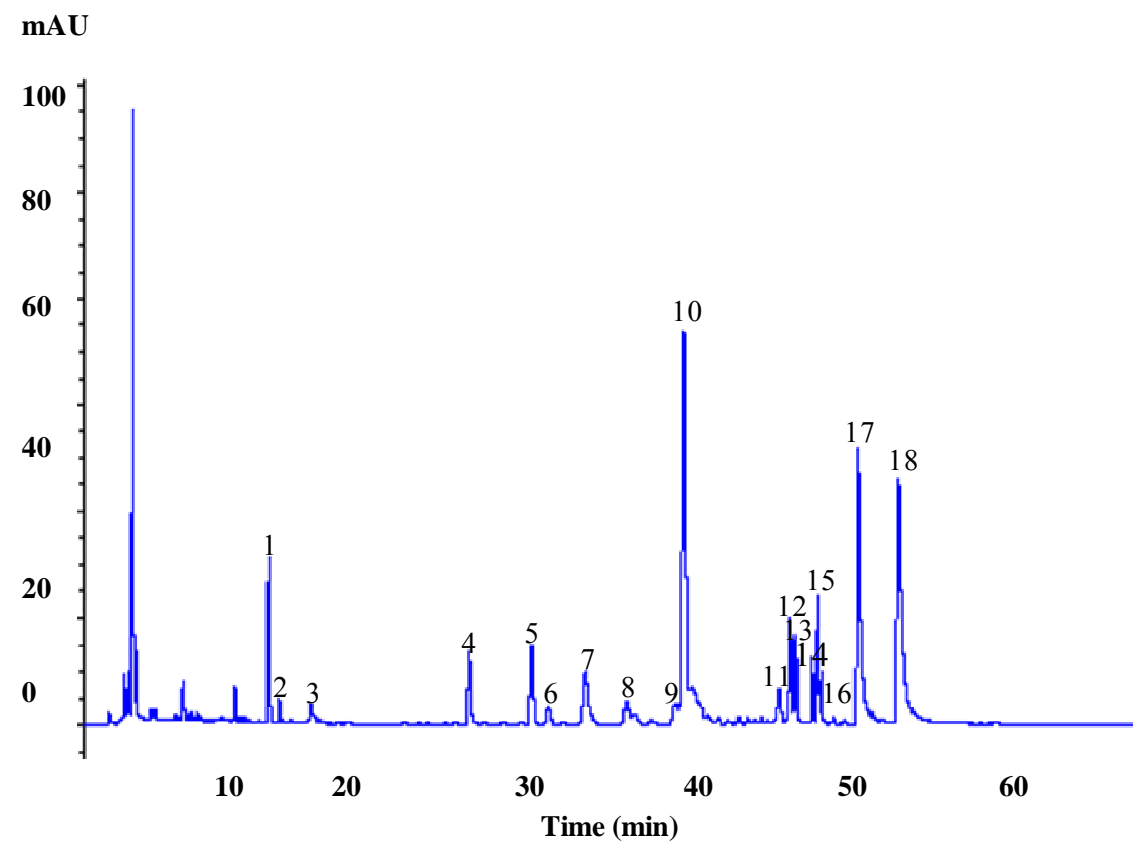

Figure 1. HPLC profiles of alkaloids isolated from the bulb of Lycoris radiata. Peak numbers refer to the alkaloids listed in Table 1: 1, unknown; 2, unknown; 3, unknown; 4, lycoricidine; 5 , unknown; 6 , hipppeastrine; 7,0 -demethyllycoramine; 8 , lycoricidinol; 9 , galanthine; 10, lycorine; 11, unknown; 12, lycorenine; 13, lycoramine; 14, galanthamine, 15, unknown, 16, homolycorine; 17, unknown; 18, pretazettine.

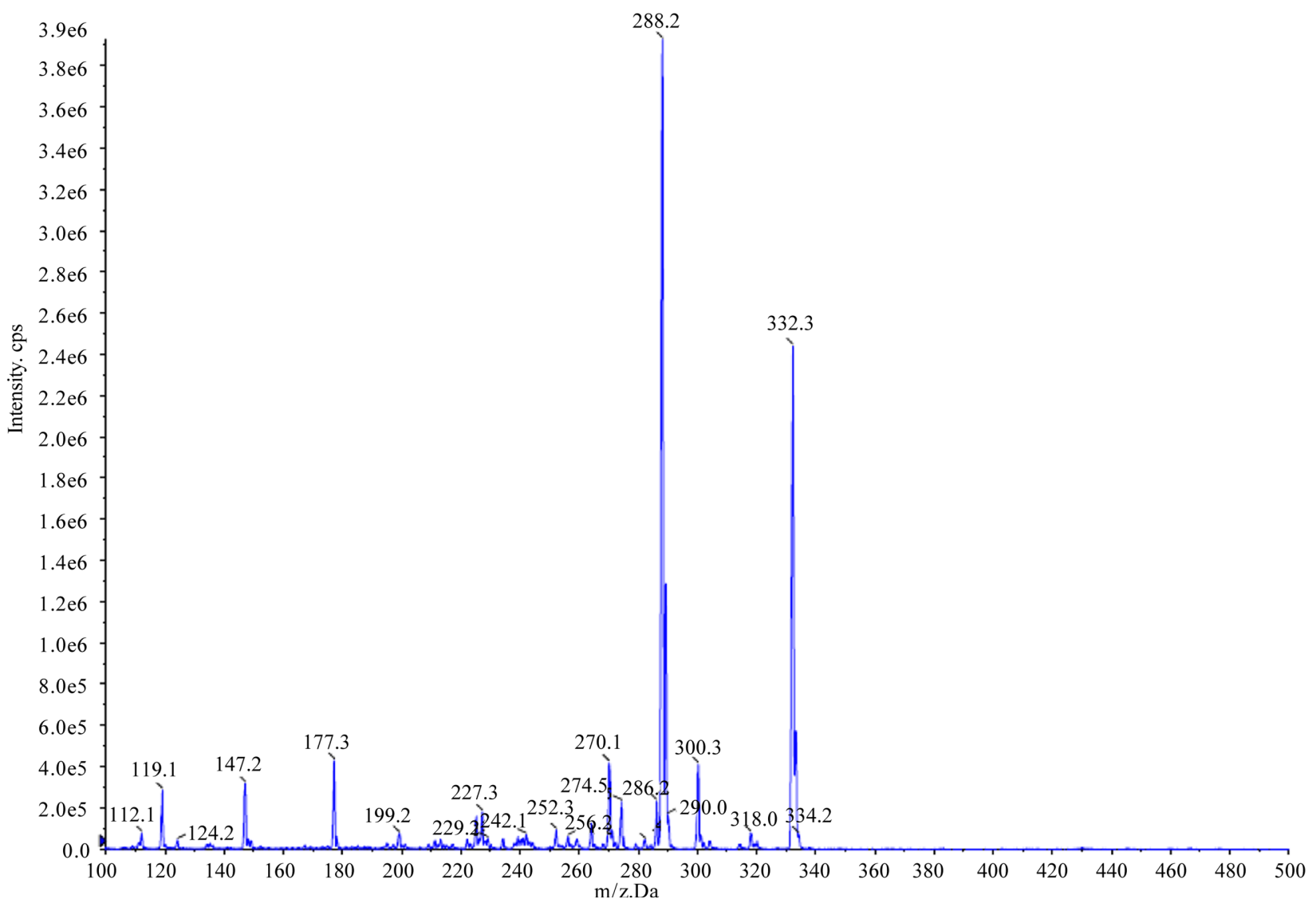

(a) 


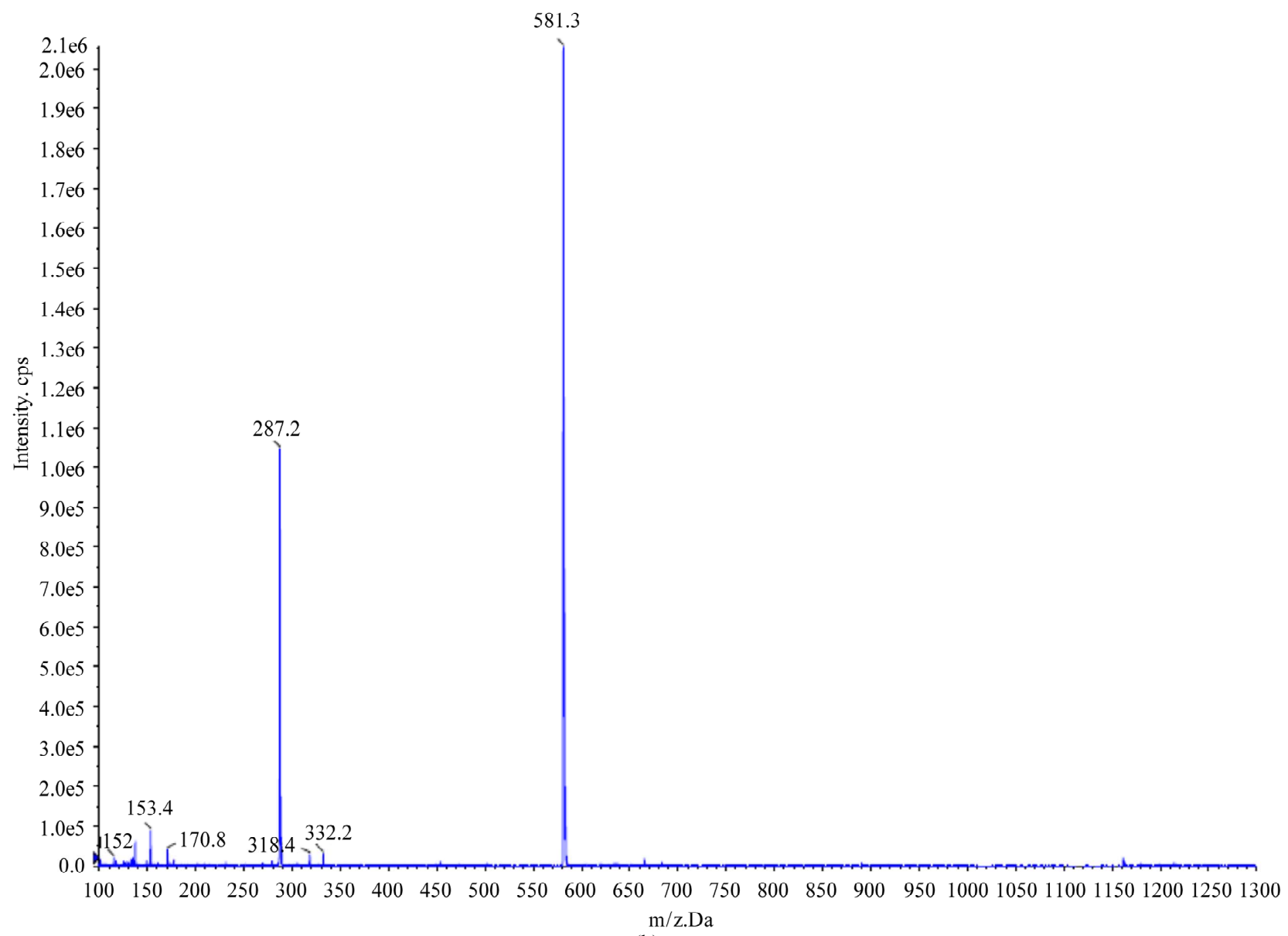

(b)

Figure 2. LC-ESI-MS spectra of (a) lycorine (alkaloid) and (b) cyanidin 3-sambubioside (anthocyanin) from Lycoris radiata.

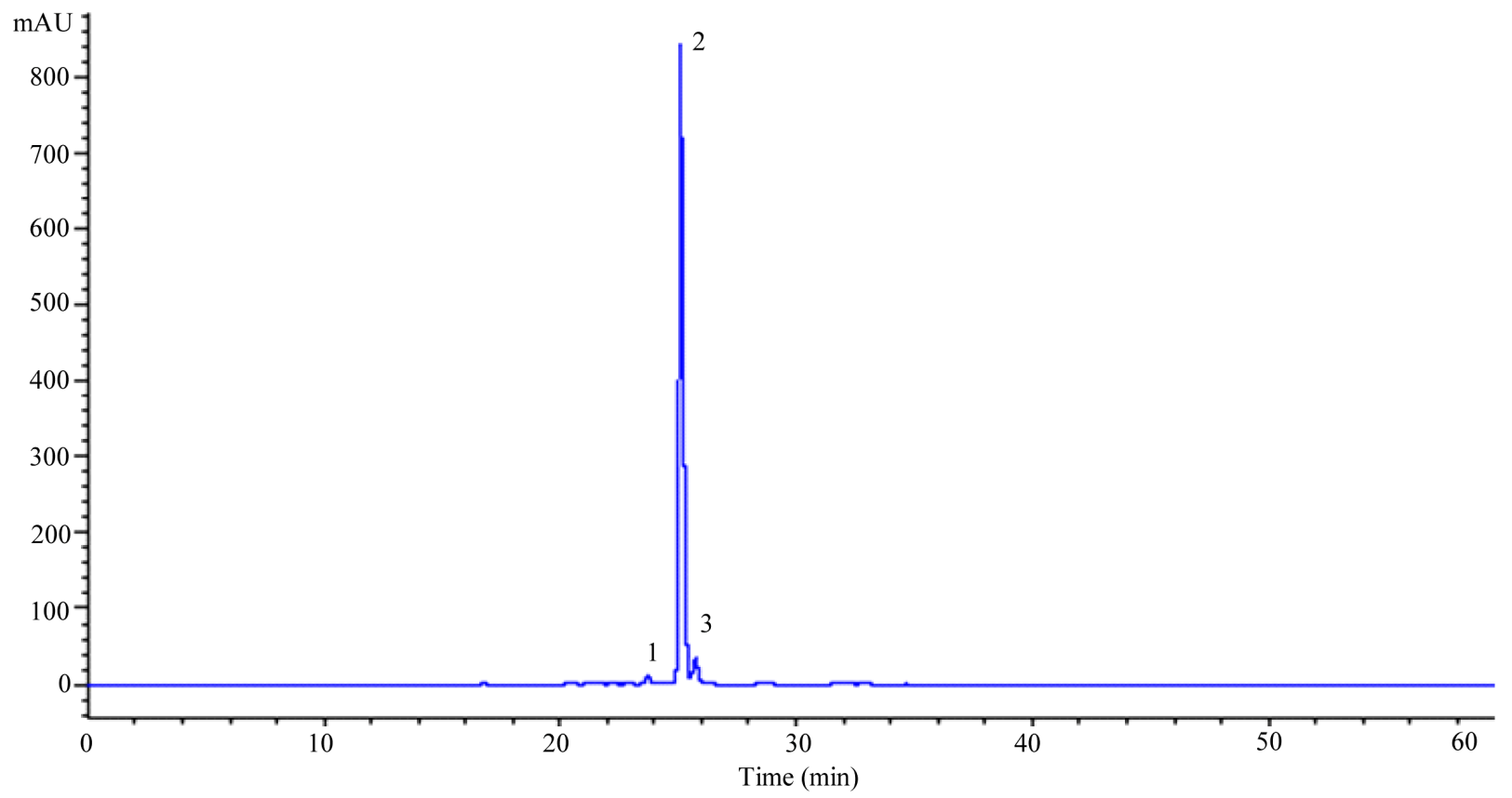

Figure 3. HPLC chromatogram of anthocyanins isolated from the flower of Lycoris radiata. Peak numbers refer to the anthocyanins listed in Table 2: 1, cyanidin 3-diglucoside; 2, cyanidin 3-sambubioside; 3, cyanidin 3-glucoside. 
Table 2. Anthocyanins identified in the flower of Lycoris radiata.

\begin{tabular}{cccc}
\hline No. $^{\text {a }}$ & RT $^{\mathrm{b}}$ & Trivial names & {$[\mathrm{M}+\mathrm{H}]^{+}(\mathrm{m} / \mathrm{z})$} \\
\hline 1 & 23.8 & cyanidin 3-diglucoside & $318 / 287$ \\
2 & 25.1 & cyanidin 3-sambubioside & $581 / 287$ \\
3 & 26.0 & cyanidin 3-glucoside & $449 / 287$ \\
\hline
\end{tabular}

${ }^{a}$ The elution order of anthocyanins in HPLC chromatogram (Figure 3). ${ }^{b}$ Retention time ( $\mathrm{min})$.

showed different retention times (23.8 vs $26.0 \mathrm{~min})$. Similar types of anthocyanins were found in red leaf lettuce, pistachio, small red bean, red cabbage and red onion respectively [8].

\section{ACKNOWLEDGEMENTS}

This research was supported by the Bio-industry Technology Development Program (Grant No. 110139-03-2-HD110), Ministry for Food, Agriculture, Forestry and Fisheries, Republic of Korea. We thank Department of Botany and Microbiology, Addiriyad Chair for Environmental Studies, College of Science, King Saud University, Riyadh, Saudi Arabia for their support.

\section{REFERENCES}

[1] Feng, T., Wang, Y.-Y., Su, J., Li, Y., Cai, X.-H. and Luo, X.-D. (2011) Amaryllidaceae alkaloids from Lycoris radiate. Helvetica Chimica Acta, 94, 178-183. doi:10.1002/hlca.201000176

[2] Zahida, I., Habib, N., Syuntaro, H. and Yoshiharu, F. (2006) Plant growth inhibitory activity of Lycoris radiata Herb. and the possible involvement of lycorine as an allelochemical. Weed Biology and Management, 6, 221-227. doi:10.1111/j.1445-6664.2006.00217.x
[3] Yosuke, T., Eri, K., Noriyuki, K., Mariko, K., Aki, I., Kazuhiko, O., Haruki, Y., Satoshi, O. and Hiromitsu, T. (2009) New lycorine-type alkaloid from Lycoris traubii and evaluation of antitrypanosomal and antimalarial activities of lycorine derivatives. Bioorganic and Medicinal Chemistry, 16, 10182-10189.

[4] Mazza, G. and Miniati, E. (1992) Anthocyanins in fruits, vegetables, and grains. CRC Press, Boca Raton.

[5] Kihara, M., Konishi, K., Xu, L. and Kobayashi, S. (1991) Alkaloidal constituents of the flowers of Lycoris radiata HERB (Amaryllidaceae). Chemical \& Pharmaceutical Bulletin, 39, 1849-1853. doi:10.1248/cpb.39.1849

[6] Zhong, J. (2005) Amaryllidaceae and Sceletium alkaloids (Review). Natural Product Reports, 22, 111-126. doi: $10.1039 / \mathrm{b} 316106 \mathrm{~b}$

[7] Wu, X. and Prior, R.L. (2005) Systematic identification and characterization of anthocyanins by HPLC-ESI-MS/ MS in common foods in the United States: Fruits and berries. Journal of Agricultural Food Chemistry, 53, 25892599. doi:10.1021/jf048068b

[8] Giusti, M.M., Rodriguez-Saona, L.E., Griffin, D. and Wrolstad, R.E. (1999) Electrospray and tandem mass spectroscopy as tools for anthocyanin characterization. Journal of Agricultural Food Chemistry, 47, 4657-4664. doi:10.1021/jf981242+

[9] Solovchenko, A.E. and Merzlyak, M.N. (2008) Screening of visible and UV radiation as a photoprotective mechanism in plants. Russian Journal of Plant Physiology, 55, 719-737.

[10] Yoo, D.S., Choi, Y.H., Cha, M.R., Lee, B.H., Kim, S.-J., Yon, G.H., Hong, K.S., Jang, Y.S., Lee, H.S., Kim, Y.S., Ryu, S.Y. and Kang, J.S.(2011) HPLC-ELSD analysis of 18 platycosides from balloon flower roots (Platycodi Radix) sourced from various regions in Korea and geographical clustering of the cultivation areas. Food Chemistry, 129, 645-651. doi:10.1016/j.foodchem.2011.04.106 